\title{
Effect of high voltage electric field on structure and property of PEDOT : PSS film
}

\author{
(C) Jinling Zhang, Juncheng Liu $\llbracket$ \\ School of Materials Science and Engineering, Shandong University of Technology, \\ 255049 Zibo, China \\ ฯ E-mail: jchliu@sdut.edu.cn
}

(Received 14.02.2017. Received after revision 30.03.2017)

\begin{abstract}
Low electrical conductivity of PEDOT:PSS film is to some extent a limit for its wide application. To solve this problem, the high voltage electric field was used to improve the film's electrical conductivity and its effects on the film's structure and properties were investigated. The PEDOT:PSS film was prepared on quartz substrate with spin coating. Visible light transmittance of the prepared film was tested with UV-Visible spectroscopy and chemical structure was measured with Fourier transform Raman spectroscopy (FTRM). The surface morphology was characterized with AFM, and electrical conductivity was measured with Hall effects measurement. The results showed that with the increase of the electric field, the electrical conductivity of PEDOT:PSS film was boosted rapidly at first, and then improved slowly when the electric field was above $200 \mathrm{kV} / \mathrm{m}$. The film's electrical conductivity improved more than 17 times in total from $0.51 \cdot 10^{-3}$ up to $8.92 \cdot 10^{-3} \mathrm{~S} / \mathrm{m}$. However, the film's visible light transmittance decreased only a little with the increase of the electric field, not more than $3 \%$. In addition, despite little change in the chemical structure of the PEDOT:PSS film, its surface roughness increased significantly with the increase of the electric field intensity.
\end{abstract}

DOI: 10.21883/FTP.2017.12.45183.8553

\section{Introduction}

Due to favorable structural stability, optical transparency and flexibility, poly (3,4-ethylene dioxythiophene): poly (styrenesulphonate) (PEDOT:PSS) has been of considerable interest and extensively studied from both the academic and technologic viewpoints [1-6]. Despite these versatile advantages, the electrical conductivity remains to be a limiting factor for its practical application in optoelectronic devices. Therefore, the improvement of the electrical conductivity of PEDOT:PSS film has been considered to be urgent and a lot of work has been done. For example, various additives such as sorbitol [7], glycerol [8], ethylene glycol [9], dimethyl sulfoxide [10], meso-erythritol [10], and poly (ethylene glycol) [11] have been added to the PEDOT: PSS solution prior to the film casting. In addition, acid treatment [12] and rinsing in water [13] were also applied to increase the film's electrical conductivity. Ouyang et al. $[13,14]$ reported that the addition of ethyleneglycol to the aqueous PEDOT: PSS dispersion could improve the electrical conductivity of the prepared film. They attributed the improved electrical conductivity to the conformational transition of the PEDOT molecular from „Benzeniod structure" to "Quinoid structure". Moreover, metal particles were also employed to improve its conductivity. Platinum nanoparticles were doped into PEDOT: PSS film to increase its current density [15]. Silver nanowires embedded PEDOT:PSS nanocomposite thin film decreased its sheet resistance more than one order of magnitude from $1.35 \cdot 10^{4}$ to $1.07 \cdot 10^{3} \Omega / \square$ [16]. Modification of PEDOT:PSS film with physical methods, such as UV treatment [17] and bias field treatment $[18,19]$ could not change the structure of PEDOT. However, the dynamic etching process greatly enhanced the conductivity of the PEDOT:PSS film [20].
Epstein et al. [21] proposed that the conducting polymer was a disordered material with free charge carriers, the mobility of which dropped with the decrease of temperature. Additionally, Zhou et al. [22] disclosed the correlation between electrical conductivity and microstructure of PEDOT:PSS film and temperature. For polar polymer molecules, the non-superposition of the cation center and the anion center creates an electric dipole moment. Oikawa et al. [23] suggested that the external electric field can polarize the dipole moment of the polar molecules, and lead to an orientation of the polymer chain. This phenomenon might improve the film's electrical conductivity.

In this work, a high voltage direct current electric field will be applied on the preparation of PEDOT:PSS film in order to improve the electrical conductivity of PEDOT: PSS film. It is suggested that when PEDOT:PSS solution is coated on the quartz substrate with the spinning disc, a vertical high voltage electric is applied on the disc. The effects of the high voltage electric field on the structure and properties of the PEDOT: PSS film will be investigated.

\section{Experiments}

Quartz flakes $\left(10 \times 10 \mathrm{~mm}^{2}\right)$ were chosen as substrates and cleaned in four steps in an ultrasonic bath for 40 minutes before spin coating. The cleaning procedure included: (a) detergent cleaning; (b) acetone cleaning; (c) ethanol cleaning and (d) deionized water cleaning. After all the four steps were carried out, the substrates were dried in a vacuum oven. Any residual organic contamination was removed with UV-ozone treatment for $30 \mathrm{~min}$.

The PEDOT:PSS aqueous solution $(1.3 \%$ dispersed in $\mathrm{H}_{2} \mathrm{O}$, from Suzhou subfamily Chemical Reagent Co., Ltd.) 


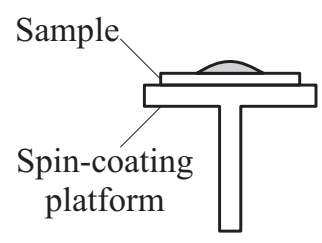

Step 1

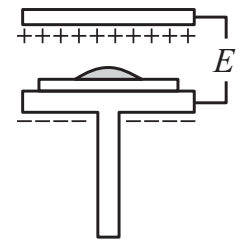

Step 2

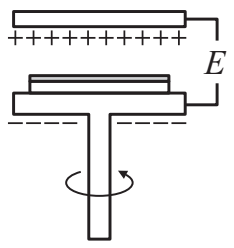

Step 3
Figure 1. The preparation of PEDOT: PSS film under vertical electric field.

was treated in ultrasonic bath for $20 \mathrm{~min}$ to make it more uniform and then filtered with a $5 \mu \mathrm{m}$ filter. As is shown in Fig. 1, the quartz substrate was fixed on a spin disk and a vertical high voltage electric field was applied on the spin disk. The solution was then dropped onto the quartz substrate rotating at $3500 \mathrm{rpm}$ for 20 seconds twice. Then the film was dried at $110^{\circ}$ for $60 \mathrm{~min}$ in a vacuum oven before any further characterization.

The molecular structure analysis was carried out with Fourier Transform Raman Spectroscopy (FTRS), which was recorded with a RFS 100 Bruker spectrometer (excitation wavelength $1064 \mathrm{~nm}$ ). The surface morphology of the PEDOT:PSS film was detected with Atomic Force Microscope (AFM), which was performed with Digital Instruments Nanoscope III operating in the tapping mode. The optical property was characterized with a TU-1901 Dual beam UV-Visible spectrophotometer. The electrical conductivity of the film was measured with Hall effects measurement system (HMS 3000). All the measurements were performed at room temperature.

\section{Results and discussion}

\subsection{Effect of high voltage electric field on the structure of PEDOT: PSS film}

Fig. 2 shows the Raman spectra of the pristine PEDOT:PSS film and the one treated with a $200 \mathrm{kV} / \mathrm{m}$ electric field. The main features of the curve $(a)$ in Fig. 2 for the pure PEDOT:PSS, are assigned as follows: 558, $989 \mathrm{~cm}^{-1}$ (oxyethylene ring deformation), $699 \mathrm{~cm}^{-1}$ (symmetric ring deformation), 1100 and $1130 \mathrm{~cm}^{-1}(C-O-C$ deformation), $1200-1280 \mathrm{~cm}^{-1} \quad\left(C_{\alpha}-C_{\alpha^{\prime}}\right.$ stretching $+C-H$ bending $)$, $1365 \mathrm{~cm}^{-1} \quad\left(C_{\beta}-C_{\beta^{\prime}}\right.$ stretching), $1424 \mathrm{~cm}^{-1}$ (symmetric $C_{\alpha}=C_{\beta}$ stretching deformation in the aromatic thiophene ring) and $1510-1580 \mathrm{~cm}^{-1}$ (antisymmetric $C=C$ stretching) [24-26]. The Raman curve principal peaks, such as $1569,1539,1256$, and $440 \mathrm{~cm}^{-1}$ are attributable to the PEDOT $[27,28]$, as shown in the curve $(a)$ in Fig. 2 . The curve $(b)$ for the modified PEDOT:PSS film is nearly the same as the curve $(a)$. In other words, the electric field did not change the chemical structure of the PEDOT:PSS film. This differs from the result from Dutta et al. [29]. They applied a direct current electric field $(75-175 \mathrm{kV} / \mathrm{m})$ next to the nozzle which sprayed the solution and found that the band between 1400 and $1500 \mathrm{~cm}^{-1}$ became narrower and red-shifted, which indicated that the thiophene ring present in PEDOT:PSS was enhanced by structural transformation from benzeniod structure to quinoid structure. The difference could be explained as follows: the size of droplet in this work was many times larger than that sprayed by a nozzle and electric fields of equal intensity could hardly affect the former, but change microstructure of the latter.

The AFM images of the PEDOT:PSS films are shown in Fig. 3. Fig. 3, a presents the pristine PEDOT:PSS

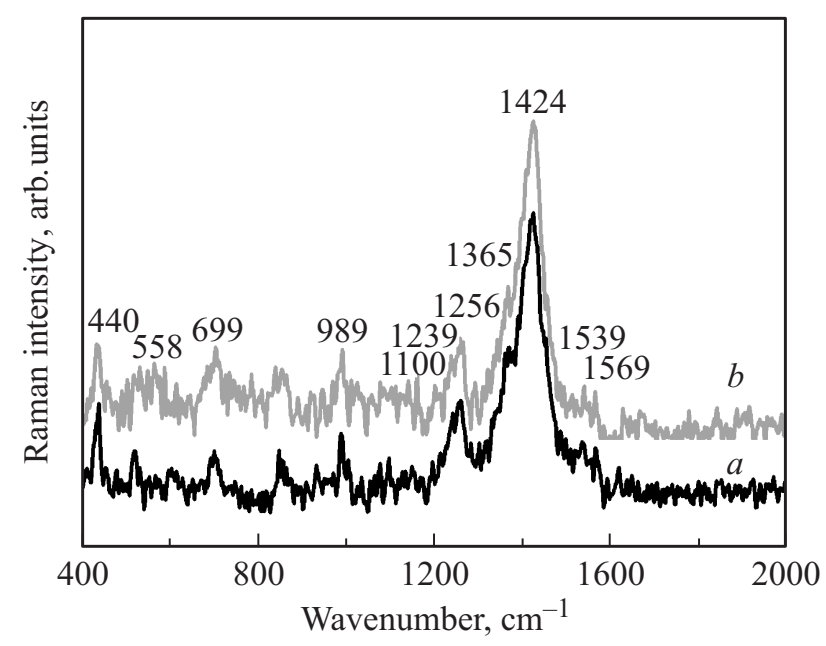

Figure 2. Raman spectra curves of the PEDOT: PSS thin films: $a$ - the pristine PEDOT:PSS film; $b$ - the PEDOT:PSS film treated with $200 \mathrm{kV} / \mathrm{m}$ electric field.
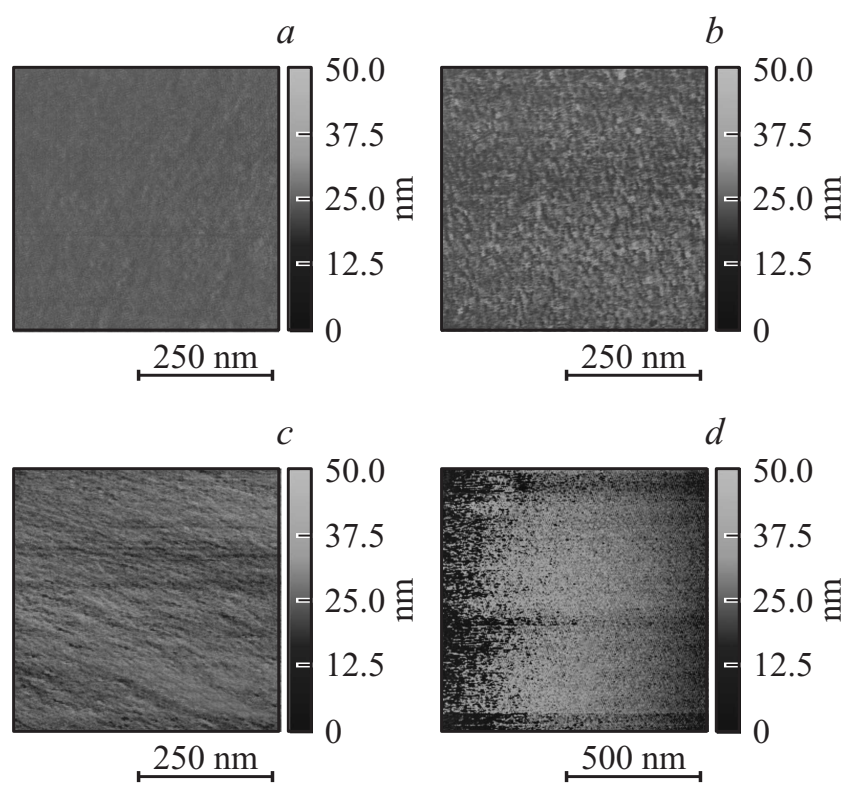

Figure 3. AFM images of PEDOT:PSS films treated with different electric field intensity: $a$ - the pristine PEDOT:PSS film, RMS roughness $1.148 \mathrm{~nm} ; b$ - with $100 \mathrm{kV} / \mathrm{m}$, RMS $2.857 \mathrm{~nm}$; $c$ - with $200 \mathrm{kV} / \mathrm{m}$, RMS $3.072 \mathrm{~nm} ; d-$ with $300 \mathrm{kV} / \mathrm{m}$, RMS $7.083 \mathrm{~nm}$. 
film. It can be seen that the surface morphology of the pristine PEDOT:PSS film is quite smooth. When PEDOT:PSS film was modified with high electric field, the roughness of the PEDOT: PSS film was obviously increased, as shown in Fig. 3, $b, c, d$. The RMS (Root mean square) roughness increased many times from 1.148 to $7.083 \mathrm{~nm}$. The higher the electric field, the larger the roughness of the PEDOT:PSS film. On the contrary, Dutta et al. [29] reported that the electric field decreased the film surface roughness significantly.

For polar polymer molecule, non-superposition of cation and anion centers creates an electric dipole moment. External electric field can polarize the electric dipole moment of polar molecule and lead to an orientation of polymer chain [23]. For PEDOT:PSS, electric dipole moments mainly constrain the grafts along the chain backbone. With spin coating, majority of the molecular chains would incline to lie on the substrate plane due to huge centrifugal force. The intense electric field enhanced the electric dipole moments made them align along the electric direction, i.e. the electric dipole moment orientation. Therefore, PEDOT:PSS molecular chains would stretch along the direction of electric field and become "longer" or "higher", and random PEDOT:PSS molecular chains become oriented to some extent. The higher the electric field, the „longer" the molecular chains. Such a change could affect the dipole structure of the surface and thereby affect the film's electrical conductivity [30,31].

\subsection{Effect of the high voltage electric field on the properties of PEDOT: PSS film}

The effect of the high voltage electric field intensity on the electrical conductivity of the PEDOT:PSS film is shown in Fig. 4. As the electric field intensity increased from 0 to $300 \mathrm{kV} / \mathrm{m}$, the electrical conductivity of the PEDOT:PSS film increased more than 17 times from $0.51 \cdot 10^{-3}$ up to $8.92 \cdot 10^{-3} \mathrm{~S} / \mathrm{m}$. Its electrical conductivity improved rapidly at first as the electric field intensity increased, and then increased slowly when the electric field intensity was above $200 \mathrm{kV} / \mathrm{m}$. Finally, the electrical conductivity remained unchanged when the electric field intensity was over $300 \mathrm{kV} / \mathrm{m}$. Such an improvement may result from the stretching and the orientation of the PEDOT:PSS molecular chains along the direction of the electric field. The similar phenomena of other polar polymer molecule films has been observed [32,33].

At first, the orientation of the electric dipole moments contributed to the intra chain charge transportation. The intense electric field could increase the delocalization of the charge and decrease the barrier of the charge transportation. Additionally, the intense electric field could increase concentration of the more conductive PEDOT by the improvement of the PEDOT: PSS ratio [28]. Moreover, there was so-called core-shell structure in the PEDOT: PSS film, where the core was conducting PEDOT rich and the shell was insulating PSS rich. The intense electric field made PEDOT and PSS

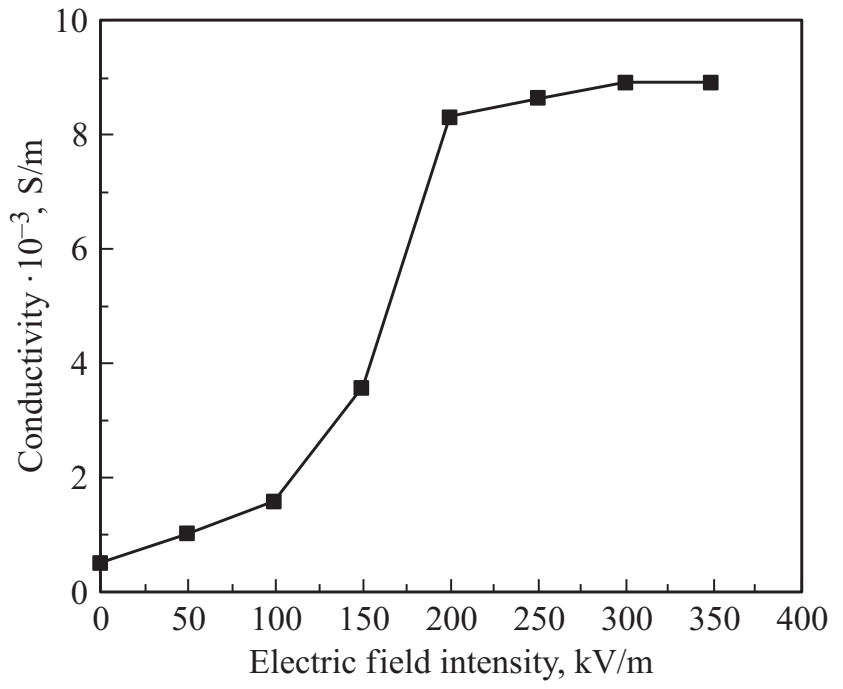

Figure 4. The effect of the electric field intensity on the electrical conductivity of the PEDOT: PSS films.

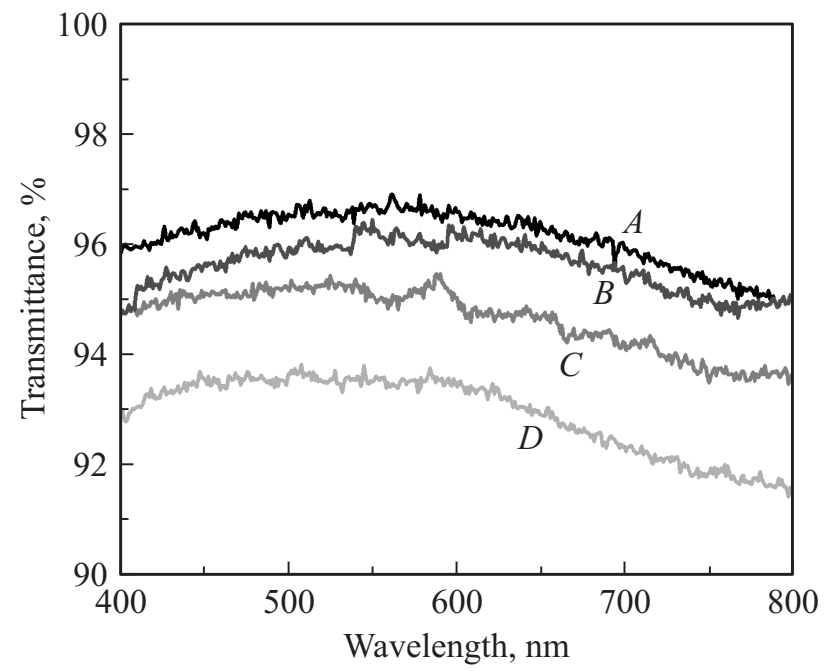

Figure 5. Effect of the electric field intensity on the transmittance of PEDOT:PSS film: $A-$ the pristine PEDOT:PSS film; $B$ - effected with $100 \mathrm{kV} / \mathrm{m}$ electric field; $C$ - effected with $200 \mathrm{kV} / \mathrm{m}$ electric field; $D$ - effected with $300 \mathrm{kV} / \mathrm{m}$ electric field.

chains redistributed, and reduced the thickness of insulating PSS shell. These changes enhanced the interchain charge transport and resulted in an interconnected network of elongated PEDOT chains, which in turn led to conductivity improvement [28]. Therefore, the electrical conductivity improved with the increase of the electric field intensity.

Transmittance plays an important role in the application of PEDOT: PSS thin film. It is always important to keep its value as high as possible at the wavelength range from 400 to $800 \mathrm{~nm}$, when PEDOT:PSS is applied on organic solar cell or organic light emitting diode devices [34]. Fig. 5 shows the transmittance of the pristine and the PEDOT:PSS films treated with the electric field. In general, the electric field slightly degraded the film's transmittance. To be 
specific, the transmittance decreased by about 1.0, 1.5, and $3.0 \%$, respectively, when the electric filed was 100 , 200 , and $300 \mathrm{kV} / \mathrm{m}$.

The degradation of transmittance may be first attributed to the increased surface roughness. Secondly, both the enhancement and the orientation of the electric dipole moments could significantly interfere with the light transmittance, as light is essentially an electromagnetic wave.

\section{Conclusion}

High voltage electric field was used to modify PEDOT:PSS film. It increased the surface roughness of PEDOT:PSS film significantly, but the chemical structure of the PEDOT:PSS film remained unchanged. In addition, while the electrical conductivity of the film was improved more than 17 times from $0.51 \cdot 10^{-3}$ up to $8.92 \cdot 10^{-3} \mathrm{~S} / \mathrm{m}$, its visible light transmittance only changed less than $3 \%$.

Acknowledgement: This work was partly supported by National Natural Science Foundation of China (Grant No. 51352002).

\section{References}

[1] S.C.J. Meskers, J.K.J. van Duren, R.A. Janssen. Adv. Funct. Mater., 13, 805 (2003).

[2] J. Jang, M. Chang, H. Yoon. Adv. Mater., 17, 1616 (2005).

[3] H. Okuzaki, M. Ishihara, S. Ashizawa. Synth. Met., 137, 947 (2003).

[4] S. Ashizawa, Y. Shinohara, H. Shindo, Y. Watanabe, H. Okuzaki. Synth. Met., 153, 41 (2005).

[5] H. Yan, S. Endo, Y. Hara, H. Okuzaki. Chem. Lett., 36, 986 (2007).

[6] H. Okuzaki, Y. Harashina, H. Yan. Eur. Polym. J., 45, 256 (2009).

[7] F. Zhang, M. Johansson, M.R. Andersson, J.C. Hummelen, O. Inganas. Adv. Mater., 14, 662 (2002).

[8] D. Bagchi, R. Menon. Chem. Phys. Lett., 425, 114 (2006).

[9] B. Yoo, A. Dodabalapur, D.C. Lee, T. Hanrath, B.A. Korgel. Appl. Phys. Lett., 90, 072106 (2007).

[10] J. Ouyang, C.W. Chu, F.C. Chen, Q. Xu, Y. Yang. Adv. Funct. Mater., 15, 203 (2005).

[11] T. Wang, Y. Qi, J. Xu, X. Hu, P. Chen. Appl. Surf. Sci., 250, 188 (2005).

[12] T.P. Nguyen, S.A. de Vos. Appl. Surf. Sci., 221, 330 (2004).

[13] J. Ouyang, C.W. Chu, Y. Yang, J. Shinar, Q. Xu, G. Li. Polymer, 45, 8443 (2004).

[14] J. Ouyang, C.W. Chu, F. Chen, Q. Xu, Y. Yang. J. Macromol. Sci., Pure Appl. Chem., 14, 1497 (2004).

[15] C.C. Chang, M.T. Jiang, C.L. Chang, C. Lan Lin. Mater. Chem. Phys., 127, 440 (2011).

[16] N. Duraisamy, S.J. Hong, K.H. Choi. Chem. Eng. J., 225, 887 (2013).

[17] A. Moujoud, S.H. Oh, K.Y. Heo, K.W. Lee, H.J. Kim. Org. Electron., 10, 785 (2009).

[18] F. Padinger, R.S. Rittberger, N.S. Sariciftci. Adv. Funct. Mater., 13, 85 (2003).

[19] Y. Li, Y. Hou, Y. Wang, Z. Feng, B. Feng, L. Qin, F. Teng. Synth. Met., 158, 190 (2008).
[20] K. Lim, S. Jung, S. Lee, J. Heo, J. Park, J.W. Kang, Y.C. Kangm, D.G. Kim. Org. Electron., 15, 1849 (2014).

[21] A.J. Epstein, F.C. Hsu, N.R. Chiou, V.N. Prigodin. Curr. Appl. Phys., 2, 339 (2002).

[22] J. Zhou, D.H. Anjum, L. Chen, X. Xu, I.A. Ventura, L. Jiang, G. Lubineau. J. Mater. Chem. C, 2, 9903 (2014).

[23] H. Oikawa, S. Fujita, H. Kasai, S. Okada, S.K. Tripathy, H. Nakanishi. Colloids Surf. A, 169, 251 (2000).

[24] S. Garreau, G. Louarn, G. Froyer, M. Lapkowski, O. Chauvet. Electrochim. Acta, 46, 1207 (2001).

[25] M. Kalbáč, L. Kavan, L. Dunsch. Compos. Sci. Technol., 69, 1553 (2009).

[26] J. Zhou, G. Lubineau. Appl. Mater. Interfaces, 5, 6189 (2013).

[27] S. Sakamoto, M. Okumura, Z. Zhao, Y. Furukawa. Chem. Phys. Lett., 412, 395 (2005).

[28] S. Garreau, G. Louarn, J.P. Buisson, G. Froyer, S. Lefrant. Macromolecules, 32, 6807 (1999).

[29] N. Chaturvedi, F. Alam, S.K. Swami, V. Dutta. J. Appl. Phys., 114, 184501 (2013).

[30] Y.J. Lin, F.M. Yang, C.Y. Huang. Appl. Phys. Lett., 91, 092127 (2007).

[31] Y.J Lin, F.M. Yang, C.S. Lin. Appl. Phys., 102, 103702 (2007).

[32] H. Jin, Y. Hou, Q. Shi, X. Meng, F. Teng. Sol. St. Commun., 140, 555 (2006).

[33] J.A. DeAro, D. Moses, S.K. Buratto. Appl. Phys. Lett., 75, 3814 (1999).

[34] B.R. Saunders, M.L. Turner. Adv. Colloid Interface Sci., 138, 1 (2008).

Редактор K.V. Emtsev 